\title{
Treatment of Melasma Cases by Oral Tranexamic Acid: A Randomized Control Trial
}

\author{
Karim ATMR ${ }^{1}$, Sadeque $\mathrm{SP}^{2}$, Ferdous $\mathrm{A}^{3}$, Khan $\mathrm{MAL}^{4}$, Rahman $\mathrm{MH}^{5}$, Rafi $\mathrm{A}^{6}$, Masum $\mathrm{A}^{7}$
}

DOI: https://doi.org/10.3329/jafmc.v14i2.45896

\begin{abstract}
Introduction: Melasma is a great challengefor a dermatologist as its treatment is unsatisfactory and recurrence is high. Treatment of melasma using oral tranexamic acid is a novel concept.
\end{abstract}

Objectives: To compare the efficacy of oral tranexamic acid with routine topical therapies for the treatment of melasma.

Materials and Methods: This prospective, interventional, randomized controlled trial was conducted among 130 melasma patients in the Department of Dermatology, Combined Military Hospital, Cumilla from June 2016 to June 2017. The patients were divided into two groups consisting of 65 patients each. The first group (Group A) was given routine treatment measures and oral Tranexamic Acid while the second group (Group B) was treated only with routine topical measures. Capsule Tranexamic Acid was prescribed at a dose of $250 \mathrm{mg}$ twice a day for three months and cases were followed during the course of treatment. The response was evaluated on the basis of Melasma Assessment Severity Index (MASI).

Results: Astatistically significant decrease in the mean Melasma Assessment Severity Index from baseline to 8th and 12th weeks was observed among group A patents $(11.08 \pm 2.91$ vs $8.95 \pm 2.08$ at week 8 and vs. $7.84 \pm 2.44$ at week $12 ;(p<0.05$ for both). While among group $B$ patients the decrease in mean score was significant at 8 weeks and insignificant at 12 weeks follow up ( $11.60 \pm 3.40$ vs $9.9 \pm 2.61$ at 8 weeks and vs. $9.26 \pm 3$ at 12 weeks; $p<0.05$ for former but $p>0.05$ for later).

Conclusion: Oral Tranexamic acid provides rapid and sustained improvement in the treatment of melasma.

Key-words: Melasma, Tranexamic acid.

\section{Introduction}

Melasma is characterized by irregular light togrey-brown macules and patches on sun exposed skin $^{1}$ commonly affecting the cheeks, forehead, upper lip, nose, chin and occasionally forearms. It is a common acquired disorder of pigmentation and is known to occur in all skin types, all ethnic groups and both sexes. It is relatively more common in darker skin type (III and IV) and more in women of childbearing age. The exact aetiology of melasmais unknown, but exposure to UV irradiation and genetic factors are considered as main causes in addition to endocrine factors (pregnancy, hormonal therapy and ovarian dysfunction), drugs (phenytoin, phototoxic drugs), cosmetics, vascular and systemic diseases like thyroid dysfunction and anaemia of multifactorial origin. These factors lead to an increased synthesis of melanosomes in melanocytes and their transfer to keratinocytes. Melasmalesions typically fade in winter and aggravate insummer. Chloasma (melasma related to pregnancy) usually diminishes within a few monthsof delivery but melasma lesions due to oral contraceptives are usually persistent ${ }^{2}$. Three clinical patterns of melasmaare recognized: malar (most common), centrofacial and mandibular ${ }^{3}$. Classification of melisma isbased on visible light, Wood's light and lesional histology which is as follows: epidermal, which has increasedmelanin predominantly in basal andsuprabasal layers of the epidermis with pigmentaccentuation on Wood's lamp. The dermal typehas perivascular melanin laden macrophages in the superficial and deep dermis and does notaccentuate with Wood's lamp. The mixedvariety has elements of both and appears as a deepbrown colour with Wood's lamp accentuation ofonly the epidermal component ${ }^{3}$. However, it is believed that usually, melasma has bothcomponents.

Treatment of melasma can be very frustrating and challenging because the aim is toobtain a decrease in melasma pigment without hypopigmentation. Till date, none of its existing treatment modalities hasprovided quick and sustained result. Today topical hydroquinone is considered to be the gold standardamong topical treatments of melasma ${ }^{4}$. Prevention ofUV radiation, topical bleaching agents, chemical peel andlight-based therapies are the currently existing plethora of remedies. Addition of tranexamicacid (TXA) for the treatment of melasma is a novel concept. Previously usedas an anti-fibrinolytic agent, TXA is

1. Lt Col A T M Rezaul Karim, MBBS, DDV, MCPS, FCPS, Associate Professor of Dermatology, AFMC, Dhaka (E-mail: drreza1031@ yahoo.com) 2. Dr Shayesta Parvin Sadeque, MBBS, DGO, Assistant Professor of Gynae\&Obs, ArmyMedical College, Cumilla 3. Dr Afreen Ferdous, MBBS, MPhil, Assistant Professor of Physiology, East-West Medical College, Uttara, Dhaka 4. Brig Gen Md Abdul Latif Khan, MBBS, DDV, FCPS, Adviser Specialist in Dermatology, CMH, Dhaka 5. Brig Md Habibur Rahman, MBBS, FCPS, Professor of Medicine, AFMC, Dhaka 6. Col Ahmed Rafi, MBBS, MPH, MPhil, Commandant, CMH, Cumilla 7. Lt Col Abdullah Masum, MBBS, DA, FCPS, Classified Specialist in Anaesthesiology, CMH, Cumilla. 
recently found to inhibitplasminogen-keratinocyte interaction decreasing thetyrosinase activity leading todecreased melanin synthesisfrom the melanocytes ${ }^{5,6}$. Very few clinical trials have been conducted regarding the efficacy of oral TXA for the treatment of melasma. This study isthus conducted to compare the efficacy of oral TXA alongwith routine treatment measures for the treatment of melasma.

\section{Material and Methods}

It is a prospective, interventional, randomized controlled trial conducted among 130 (87 females and 43 males) melasma patients in the Department of Dermatology, Combined Military Hospital, Cumilla from June 2016 to June 2017. This study was conducted after taking authorization from the institutional review committee. Patients age ranged from 17-55 years andwere checkedfor bleeding time, clotting time, platelet count beforethey were included in the study. Patients with abnormal parameters and patients who discontinued the treatment or wanted chemical peel or laser for better response were excluded from the study. The type ofmelasma was graded with Wood's lamp and dermatoscopic examination. Patients were randomly divided into two groups consisting of 65 patients each using a random number table. The first group (Group A) was added on with oral TXA along with routine topical treatment measures while the second group (Group B) was treated only with routine topical measures. Topical treatment included topical hydroquinone and sunscreen according to the skin types. Capsule TXA ((Trans-4-aminomethyl cyclohexanecarboxylic acid)) was prescribed at adose of $250 \mathrm{mg}$ twice a day for three months and cases were followed up every four weeks till the end of the third month.The response was evaluated on the basis of Melasma Assessment Severity Index (MASI) score ${ }^{7}$. MASI score was calculated during the start of therapy (baseline) and at the end of 8th and 12 th weeks.

MASI is a scoring system introduced by Kimbrough-Green CK et al used to quantify the severity of melasma ${ }^{7}$. Four areas of the face are evaluated: forehead $(F)$, rightmalar region $(M R)$, left malar region(ML) and chin(C), corresponding to $30 \%$, $30 \%, 30 \%$, and $10 \%$ of the total facerespectively. Amount of pigmentation involved by melasmain these four areas (AF, AMR, AML, and AC) is graded asa numerical value: 0 : no involvement; 1: less than 10\% involvement; 2 : $10-29 \%$; 3: $30-49 \%$; 4: $50-69 \% ; 5: 70-89 \%$ and $6: 90-100 \%$. The severity of melasma is graded upon two factors; darkness (D) of melasma compared to the normal skin and homogeneity $(\mathrm{H})$ of hyperpigmentation. These are assessed on a scale from 0 to 4 . The rating scale for both darkness and homogeneity of melasma is as follows; 0 :absent; 1 : slight; 2 : mild; 3 : marked; and 4: maximum. MASIscore is then calculated according to the following formula:MASI $=0.3(\mathrm{DF}+\mathrm{HF}) \mathrm{AF}+0.3(\mathrm{DMR}+$ $\mathrm{HMR}) \mathrm{AMR}+0.3(\mathrm{DML}$
$+\mathrm{HML}) \mathrm{AML}+0.1(\mathrm{DC}+\mathrm{HC}) \mathrm{AC}$.The maximum score for MASI can be 48 while the minimum canbe 0 . SPSS 16 was used for the statistical analysis. Mean MASI scores were compared with students' T-test and statistical significance was compared. Patient satisfaction score was subjectively graded on the basis of a four-pointLikert scale: excellent, good, fair, and poor. Possible side effects of TXA were noted on follow up of patients.

\section{Results}

Among the study participants $(n=130)$, both groups Group A $(n=65)$ and Group B $(n=65)$ had the majority of female participants $44(67.69 \%)$ and $43(66.15 \%)$ respectively. In Group A patients, epidermal melasma was seen in 47(72.31\%) followed by mixed type 11 (16.92\%) and dermal 07 (10.77\%). In Group B patients, the majority had epidermal melasma 40 (61.54\%) followed by mixed type 18 (27.69\%) and dermal 07 (10.77\%). Distribution of melasma in Group A was frontal 07 (10.77\%), centrofacial $51(78.46 \%)$, chin $07(10.77 \%)$ and in Group B, frontal 07 (10.77\%), centrofacial 54 (83.07\%), chin 04 (6.15\%) respectively (Table-I). In Group A patients, 36.90\% of participants showed Good and $45.40 \%$ showed Excellent improvement and the satisfaction score was $11.08 \pm 2.91$ at baseline vs $8.95 \pm 2.08$ at week 8 and $7.84 \pm 2.44$ at week 12 (Figure-1). Group B patients, $40 \%$ of participants showed Satisfactorily and $32.30 \%$ showed Good improvement and the satisfaction score was $11.60 \pm 3.40$ at baseline vs $9.9 \pm 2.61$ at 8 weeks and vs. $9.26 \pm 3$ at 12 weeks (Figure-2). The improvement in Group A patients at 8th and 12th week was statistically significant $p$-value $<0.05 \%$ but in Group B patients, findings at 8 th week only were statistically significant.

Table-I: Gender of patents, pathological type and distribution of melisma $(\mathrm{N}=130)$

\begin{tabular}{|l|l|c|c|c|c|}
\hline \multirow{2}{*}{ Clinical Profile } & \multicolumn{2}{c|}{ Group A } & \multicolumn{2}{c|}{ Group B } \\
\cline { 3 - 6 } & Male & $n=65$ & $\%$ & $n=65$ & $\%$ \\
\hline \multirow{2}{*}{ Gender } & Female & 41 & 32.30 & 22 & 33.85 \\
\hline \multirow{3}{*}{ Type of Melasma } & Epidermal & 47 & 67.69 & 43 & 66.15 \\
\cline { 2 - 6 } & Dermal & 07 & 10.71 & 40 & 61.54 \\
\cline { 2 - 6 } & Mixed & 11 & 16.92 & 18 & 10.77 \\
\hline \multirow{3}{*}{ Distribution } & Frontal & 07 & 10.77 & 07 & 10.77 \\
\cline { 2 - 6 } & Centrofacial & 51 & 78.46 & 54 & 83.07 \\
\cline { 2 - 6 } & Chin & 07 & 10.77 & 04 & 6.15 \\
\hline
\end{tabular}

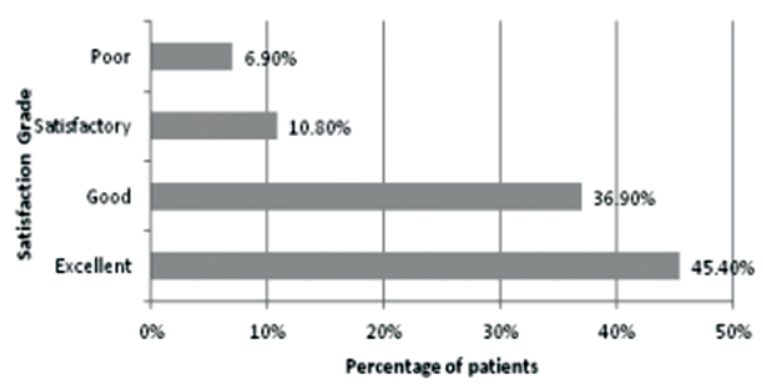

Figure-1: Satisfaction scores for Group A patients at 12 weeks 


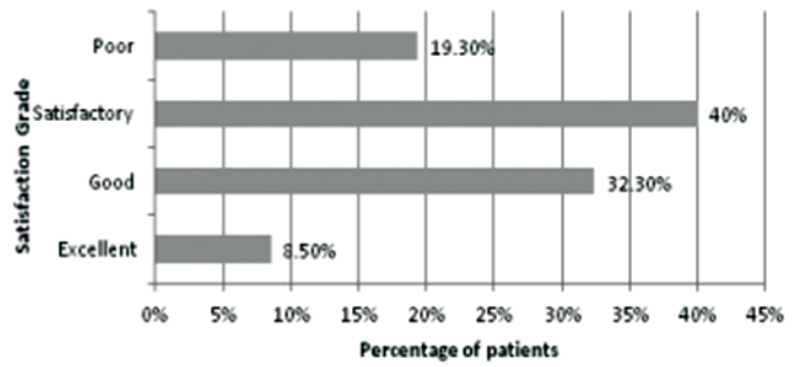

Figure-2: Satisfaction score for Group B patients 12 weeks

\section{Discussion}

Clinical trials have shown that topical medications demonstrate some efficacy in the epidermal type but not inthe dermal or mixed type of melasma ${ }^{8,9}$. Prolonged application, slow response, limited effects and undesirable recurrence are the major disadvantages of topical therapies causing patients to abandon the treatment. Also, topical bleaching agents may irritate the skin and develop post-inflammatory hyperpigmentation or result in exogenous ochronosis. Laser-assisted treatment is a good method however recurrence remains disappointng ${ }^{10}$. The most effective and safe treatment for melasma is thus yet to be explored. TXA is used as a haemostatic agent due to itsantifibrinolytic acton ${ }^{11}$. It is a synthetic derivative of the amino acid lysine used to treat and prevent excessive bleeding. Nijor $\mathrm{T}^{12}$ first studied and reported the action of TXA on melasma in 1979. However, limited studies are found in the literature regarding the use of TXA onmelasma. Maeda $\mathrm{K}$ et al studied the role of TXA inhuman melanocyte and keratinocyte cultures ${ }^{11,12}$. Their results revealed that TXAinhibits melanin synthesis inepidermal melanocytes' tyrosinase activity by blocking the interaction of melanocytes and keratinocytes by inhibition of plasminogen/ plasmin system. TXA acts by attaching tothe lysine-binding sites of plasmin and plasminogen and also prevents ultraviolet rays induced pigmentation.

Similar study as this one was conducted on a split-face based trial with topical TXA gel ${ }^{13}$. Their result showed lightening of pigmentation but was insignificant in comparison to the control group. Also, topical TXA produced erythema. Lee JHet al injected localized intradermal microinjection of TXA for melasma and found statistically significant lightening ${ }^{9}$. Histological evaluation following oral and topical TXA formelasma was done which concluded that TXA decreases epidermal pigmentation associated with melasma and also reversesmelasma-related dermal changes, such as vessel number and increased numbers of mast cells ${ }^{14}$. Uses of oral TXA for melasma in similar dose have been tried in Chinese population and authors recommend TXA to be an effective and safe therapy for the treatment of melasma ${ }^{15}$. The dose of oral TXA used in melasma is far less than that prescribed for its haemostatic action. Venous thromboembolism, myocardial infarction, cerebrovascular accidents and pulmonaryembolism are some reported complications in a haemostatic dose of
TXA. It is contraindicated for patients having acquired defective colour vision, an active intravascular clotting condition, and hypersensitivity to TXA. Hence, proper patent selection ruling out anyrisk factor resulting in hypercoagulability is of pivotal importance prior to the start of therapy.

The results of this study are consistent with the results of related studies where successful lightning was observed following oral TXA administration. Despite the lack of objective measurement in the improvement, a good clinical outcome of melasma was observed. Longer duration follow-up and randomized controlled trials involving a larger number of patients are recommended for further exploration of the efficacy of TXA in the near future. Oral TXA being a non-invasive, non-irritating drug with low side effect profile, can be routinely recommended in low dose to the patients with melasma.

\section{Conclusion}

Addition of oral TXA to the routine treatment measures provides a rapid and better lightening in patients withmelasma. Low dose oral TXA is thus recommended for the treatment of melasma.

\section{References}

1. Anstey AV. Disorders of Skin Color. In: Burns T, Breathnach S, CoxN, Grifths C eds. Rook's Textbook of Dermatology. 8th ed. Wiley Blackwell 2010:34-58.

2. Katsambas AD, Stratgos AJ, Lot TM. Melasma. In: Andreas DK, Lot TM eds. European Handbook of Dermatological Treatments. 2nd ed. Springer 2003:336.

3. Karn D, Khatri R, Timalsina M. Prevalence of skin diseases in Kavre district, Nepal. Nepal Journal of Dermatology, Venereology \& Leprology 2010; 9(1):7-9.

4. Karn D, Kc S, Amatya A et al. Oral tranexamic acid for the treatment of melasma. Kathmandu University Medical Journal 2012; 10(4):40-3.

5. Maeda K, Tomita Y. Mechanism of the inhibitory effect of tranexamic acid on melanogenesis in cultured human melanocytes in the presence of keratinocyte-conditioned medium. Journal of health science. 2007; 53(4):389-96.

6. Maeda K, Naganuma M. Topical trans-4-aminomethylcyclohexanecarboxylic acid prevents ultraviolet radiation-induced pigmentation. Journal of Photochemistry and Photobiology B: Biology. 1998; 47 (2-3):136-41.

7. Kimbrough-Green CK, Griffiths CE, Finkel LJ et al. Topical retinoic acid (tretinoin) for melasma in black patients: A vehicle-controlled clinical trial. Archives of Dermatology 1994; 130(6):727-33.

8. Ejaz A, Raza N, Iftikhar N, Muzzafar F. Comparison of 30\% salicylic acid with Jessner's solution for superficial chemical peeling in epidermal melasma. Journal of the College of Physicians and Surgeons--Pakistan: JCPSP 2008; 18(4):205-8. 
9. Lee JH, Park JG, Lim SH et al. Localized intradermal microinjection of tranexamic acid for treatment of melasma in Asian patients: A preliminary clinical trial. Dermatologic Surgery 2006; 32(5):626-31.

10. Karn D, KC S, Amatya A et al. Q-Switched Neodymium-Doped Yttrium Aluminum Garnet laser therapy for pigmented skin lesions: Efficacy and safety. Kathmandu University Medical Journal 2012; 10(2):46-50.

11. Dunn CJ, Goa KL. Tranexamic acid. Drugs 1999; 57(6):1005-32.

12. Nijor T. Treatment of melasma with tranexamic acid. Clin Res 1979; 13(13):3129-31.
13. Kanechorn Na Ayuthaya P, Niumphradit N, Manosroi Aet al. Topical $5 \%$ tranexamic acid for the treatment of melasma in Asians: a double-blind randomized controlled clinical trial. Journal of Cosmetic and Laser Therapy 2012; 14(3):150-4.

14. Na Jl, Choi SY, Yang SH et al. Effect of tranexamic acid on melasma: A clinical trial with histological evaluation. Journal of the European Academy of Dermatology and Venereology 2013; 27(8):1035-9.

15. Wu S, Shi H, Wu Het al. Treatment of melasma with oral administration of tranexamic acid. Aesthetic Plastic Surgery 2012; 36(4):964-70. 